\title{
SDTrimSP-2D studies of the influence of mutual flux arrangement on erosion and deposition
}

\author{
A. Mutzke ${ }^{(a)}$, R. Schneider ${ }^{(a)}$, I. Bizyukov ${ }^{(b)}$. \\ ${ }^{(a)}$ Max-Planck-Institut für Plasmaphysik, EURATOM Association, Wendelsteinstr. 1, 17491 \\ Greifswald, Germany \\ ${ }^{(b)}$ University of Toronto Institute for Aerospace Studies, 4925 Dufferin Street, Toronto, \\ Ontario, Canada M3H 5 T6
}

\begin{abstract}
In this work simultaneous bombardment of a nano-scale rough tungsten surface with $6 \mathrm{kV}$ carbon and $3 \mathrm{keV}$ deuterium ions is investigated by simulations with SDTrimSP and SDTrimSP-2D and compared with experiments. For same bombardment conditions a rough surface produces higher sputter yields and at the same time higher areal densities of implanted $\mathrm{C}$ than in the case of a smooth surface. For co-axial flux arrangement bombarding a rough surface under $45^{\circ}$, the surface tends to prevent $\mathrm{W}$ sputtering by aligning the surface profiles to the perpendicular and parallel directions of the impinging $C$ and $\mathrm{D}$ ion fluxes.
\end{abstract}

PACS: 61.80.Jh, 68.03.Hj, 68.37.Hk, 68.37.Ps

JNM keywords: Tungsten, Deuterium, Carbon, Ion Irradiation.

PSI-18 keywords: tungsten, carbon impurities, deuterium inventory, sputtering.

*Corresponding author address: Ralf Schneider, Max-Planck-Institut für Plasmaphysik, EURATOM Association, Wendelsteinstr.1, D-17491 Greifswald

*Corresponding author E-mail: ralf.schneider@ipp.mpg.de

Presenting author: Ralf Schneider

Presenting author e-mail: ralf.schneider@ipp.mpg.de 


\section{Introduction}

The inner wall of the vacuum vessel of the International Tokamak Experimental Reactor (ITER) will be constructed of three different materials exposed to high recycling particle fluxes [1, 2, 3]. Tungsten (W) coating will cover the divertor baffle region and possibly the first wall. It is expected that the recycling flux bombarding the tungsten surface will be seeded by carbon (C) atoms and ions. C ions are accelerated by the sheath potential towards the in-vessel surface up to energies of several keV [4]. This leads to both sputtering of $\mathrm{W}$ by energetic particles and formation of mixed $\mathrm{W}-\mathrm{C}$ surface due to $\mathrm{C}$ ion implantation.

Multi-species bombardment with C and D ions can lead to either surface erosion or C layer deposition. Prediction of the scenario can be done by binary collision approximation (BCA) based Monte-Carlo codes like SDTrimSP [5]. Typically, they deal with perfectly smooth surfaces. However, the surface morphology is a key factor in sputtering and deposition processes, mostly ignored in this approach. In order to consider effects related to surface morphology, the code SDTrimSP-2D was developed [6]. Its application [6] has shown that it is capable to provide qualitative 2D results to describe the evolution of $3 \mathrm{D}$ structures on a W surface under $\mathrm{C}$ ion bombardment. It allows access to details of the ionsurface interactions like local sputter yields, which are not available by experimental diagnostics.

One of the factors, which introduce non-flat surface morphology, is linked with the mutual flux arrangement and its effect on surface condition. Indeed, for a 1-D case (the depth dimension) or just a flat surface, only the incidence angle of each ion species is important. If lateral effects are considered, in addition to the angle of incidence of the different species also their specific geometrical arrangement defines whether the surface is eroded or is covered by a deposited $C$ layer.

This paper continues the work, which has been begun in [6]. The effect of simultaneous bombardment of rough W surface with $6 \mathrm{keV} \mathrm{C}$ and $3 \mathrm{keV}$ D ions is studied by simulations with SDTrimSP-2D. The C-fraction in the total incident flux was used in simulations to be $15 \%$. The ion energies and C-fraction exceed typical ones for ion-surface interactions in fusion. High energies and $C$ fractions have been chosen to provide high rates of $\mathrm{W}$ erosion allowing to observe the modification of the $\mathrm{W}$ surface morphology within reasonable calculation times of several days. Both ion species irradiate the surface at angles of incidence of 15 and $45^{\circ}$ to the normal of the macroscopic plane. The surface was characterized with AFM measurements identifying regions with different roughness. Fluxes are arranged symmetrically $\left(+15\right.$ or $45^{\circ}$ and -15 and $45^{\circ}$ ) and co-axially (both fluxes incident at +15 and $45^{\circ}$ ). The differences for the different geometries as well as differences between 1D and 2D simulations are discussed.

\section{The physical model implemented in SDTrimSP-2D}

SDTrimSP-2D is based on the BCA model, which is implemented in well known codes of the TRIM family. This model allows to calculate ion transport in amorphous materials, formation of collision cascades and related effects. Bombardment of a W surface with $\mathrm{C}$ ions will lead to formation of the mixed material layer. The dynamic version of the model (implemented in SDTrimSP) also allows to calculate fluence dependent elemental composition of the surface and 1D (depth dimension) elemental distribution below the surface. Experimental validation of the model was achieved for bombardment of a smooth W surface with C ions [7] and with C and He ions simultaneously [8]. 
At the same time, it has been found that a 1-D model fails to handle a surface with pronounced roughness [7,9]. The newly developed SDTrimSP-2D code is a further extension of the 1D SDTrimSP model resolving a second (lateral) dimension. This provides the opportunity to study effects of surface morphology on sputtering and implantation processes and vice versa. The code allows monitoring of the dynamic modification of the 2D surface morphology and elemental composition under ion bombardment and provides the tools for studying local parameters of ion-surface interactions. This helps to understand the macroscopic results as a consequence of surface geometry on the nano scale and its impact on local parameters to which there is no direct experimental access [6].

The model still has certain limitations for the given projectile-surface combination. Chemical reactions, which may affect the surface composition, are not considered. However, the methane production (result of $\mathrm{C}$ chemical erosion) in the given system at ITER relevant temperatures is an order of magnitude lower than that for pure $C$ (if it exists at all) [10]. Diffusion of C into the bulk of W surface at temperatures above $1073 \mathrm{~K}$ [11] is also excluded from the model.

\section{Results and discussion}

Figure 1 shows the fluence dependent of the $\mathrm{W}$ sputter yield and $\mathrm{C}$ areal density as obtained by $1 \mathrm{D}$ and $2 \mathrm{D}$ simulations and experimental data for the $15^{\circ}$ case. The corresponding evolution of the 2D surface morphology, as well as its initial state is shown in Figure 2. Two characteristic cases of the experimental surface profiles are used in the 2D studies, marked in the following as 'small roughness' and 'large roughness'. As can be seen from Figure 2 the 2D simulation results for the $15^{\circ}$ cases and the different roughness are able to reproduce the experimental range, whereas 1D approximations with modified average angles of incidence are not at all capable of that.

\subsection{Perfectly smooth surface (1D simulations)}

The evolution of the $\mathrm{C}$ areal density and the $\mathrm{W}$ sputter yield calculated for the $1 \mathrm{D}$ case turns into the regime of steady-state erosion: after a fluence of $1-2 \times 10^{22} \mathrm{~m}^{-2}$ the $\mathrm{C}$ areal density (see Figure $1(\mathrm{a}, \mathrm{c})$ ) and W sputter yield (Figure 1 (b)) both reach steady state values. At these conditions, there is a mixed-surface layer, containing $\mathrm{W}, \mathrm{C}$ and $\mathrm{D}$ atoms. The layer composition after reaching steady-state remains unchanged. It has been found in [12] that at an angle of incidence larger than $40^{\circ}$ bombardment of a W surface with $6 \mathrm{keV}$ $\mathrm{C}$ ions leads to continuous net erosion. The addition of $\mathrm{D}$ ions flux does not change this regime.

Simultaneous bombardment for the same parameters, but at an angle of incidence of $15^{\circ}$ leads to deposition of the C-D layer, which protects the $\mathrm{W}$ surface from further sputtering [9]. Its evolution is characterized by the monotonous decrease of the $\mathrm{W}$ sputter yield and at the same time an increase of the $\mathrm{C}$ areal density.

\subsection{Surface with small roughness}

The evolution of a $\mathrm{W}$ surface with small roughness at the same conditions behaves qualitatively similar (Figure 1). The steady-state is reached at a fluence, which is twice higher than that for the $1 \mathrm{D}$ case. One may note the difference between symmetrical and coaxial bombardment in the fluence range up to $5 \times 10^{22} \mathrm{~m}^{-2}$. It appears due to the geometrical factors (local angle of ion incidence and local flux ratio). After that fluence, the steadystate is reached in both cases. The resulting steady-state areal density and W sputter yield 
values are quite close to those achieved for 1D simulation. In fact, one would not be able to distinguish it experimentally, because even in a well controlled laboratory environment the typical scatter of sputter yield values for W bombarded by light ions is about $100 \%$ (see recent overview of experimental and calculation results in [13, p.87-88]).

However, there is a noticeable difference between the evolutions of a perfectly smooth surface and a surface even with small roughness: the surface with a small roughness is able to provide both an increase of the $\mathrm{W}$ sputter yield and areal density of the implanted $\mathrm{C}$ at the same time in comparison to the smooth surface.

These results can be understood if one considers the evolution of the surface morphology shown in Figure 2 (a) and (b). The surfaces, which are close to normal to the incidence direction of the $\mathrm{C}$ ion flux, tend to be covered by a $\mathrm{C}$ layer. These surfaces provide a $\mathrm{C}$ areal density higher than that for the smooth surface. Inversely, the surface under bombardment with $\mathrm{C}$ ion flux at tilted angles is eroded faster, because moment transfer from $\mathrm{C}$ to $\mathrm{W}$ atom is more effective than from $\mathrm{D}$ (due to the mass difference). The contribution of this area to the $\mathrm{W}$ sputter yield is higher than that for the smooth surface. Therefore, lateral non-uniformity of the surface increases both erosion rate and $\mathrm{C}$ implantation at the same time.

It is interesting to note that the ion bombardment practically does not change the roughness level for the $45^{\circ}$ case; it just shifts the roughness in the lateral direction. It is difficult to judge, whether the surface profile will be self-supporting at higher fluences. This issue will be addressed in future simulations.

\subsection{Surface with large roughness}

In the case of larger roughness, the regime of ion-surface interactions (erosion or deposition) changes with mutual flux arrangement, especially visible for the $45^{\circ}$ geometry. Areal densities of implanted $C$ grow at both arrangements (see Figure 1 (a)) faster and reach larger values than that for the smooth and low-roughness surfaces. This is because more parts of the surface are now close to normal to the incidence direction of the $\mathrm{C}$ ion flux. Such surface parts are more suitable for the $C$ layer deposition.

At the same time, the $\mathrm{W}$ sputter yield tends to decrease monotonically for the case of co-axial flux arrangement (Figure 1 (b)). The corresponding change of the surface morphology is shown in Figure 2 (d). All areas of the surface tend to segregate into two types: one perpendicular to the $\mathrm{C}$ and $\mathrm{D}$ flux direction and one parallel to both of them. The surface areas of the first type are covered with C-D deposited layers because of the ineffective $C$ removal at normal incidence [9]. The surface parts of the second type are effectively sputtered until they get shadowed by getting practically parallel to the $C$ and $D$ ion fluxes. This results in a decrease of the $\mathrm{W}$ sputter yield in these parts down to practically 0 and a growth of the $C$ layer on this part of the surface at higher fluence.

In case of the symmetrical flux arrangement, the $\mathrm{W}$ sputter yield initially decreases up to a fluence of $3.5 \times 10^{22} \mathrm{~m}^{-2}$ and then starts to increase. It reaches steady-state at a higher fluence similar to the case of co-axial flux arrangement for low roughness level.

\section{Comparison with experiments}

Figure 1 (c) contains also experimental results obtained for the given target-projectile combination and the same energies, but the incidence angle was $15^{\circ}$ and $\mathrm{C}$ fraction in total incident flux was slightly varied in the range of $13.4 \%-16.5 \%$ [9]. These bombardment conditions were simulated and experimental data were compared to the simulation results to verify how realistic they are.

The 2D simulations demonstrate nicely that these simulations quite naturally recover 
the experimentally observed range of values by using the two cases of small and large roughness. Obviously, the $\mathrm{W}$ surface has areas with different roughness characteristics. Experimental data are conditioned by the integral characteristics of surface roughness, which vary usually for each particular surface. In experiment, one may measure all the different values from small to large roughness resulting in a relatively large scatter in the experimental data sets. Using 1D simulations, one can partly reproduce the 2D results by changing the effective angle of incidence, but this procedure is not able to get consistent results for the two different geometries of symmetric and co-axial bombardment. Further details of the experimental work and accompanying studies will be published soon.

\section{Conclusions}

Simulations on simultaneous bombardment of a W surface with $6 \mathrm{keV} C$ and $3 \mathrm{keV}$ D ion fluxes were performed using SDTrimSP (1D case for a perfectly smooth surface) and SDTrimSP-2D codes (2D cases for small and large roughness). The 1D simulation predicts that for an angle of incidence of $45^{\circ}$ the surface is continuously eroded by the ion fluxes. In the case of a rough surface, marginally higher sputter yields and higher areal densities of implanted $\mathrm{C}$ have been observed. Such simultaneous increase of the sputter yield and $\mathrm{C}$ implantation is possible due to lateral non-uniformity of the surface and geometrical factors (local incidence angles). Parts of the surface with increased local angles of $\mathrm{C}$ ion incidence are sputtered more effectively. Parts perpendicular to the $\mathrm{C}$ ion flux are more prone to $\mathrm{C}$ deposition. In case of co-axial flux arrangement, the rough surface tends to prevent $\mathrm{W}$ sputtering by re-arranging the surface profile into parts, which are almost perpendicular and parallel to the $\mathrm{C}$ and $\mathrm{D}$ ion flux.

\section{Acknowledgements}

Ralf Schneider acknowledges funding of the work by the Initiative and Networking Fund of the Helmholtz Association. 


\section{References}

[1] G. Janeschitz, ITER JCT and ITER HTs, J. Nucl. Mat. vol. 290-293 p.1 (2001).

[2] D. Meade, et al., Mission and design of the fusion ignition research experiment, in: Proceedings of the 18th IAEA Conf. on Fusion Energy, Sorrento, Italy October, 2000, (CD-ROM), pp. IAEA-CN-77/FTP2/16, IAEA, Vienna, 2001.

[3] S. Nishio, et al., Conceptional design of advanced steady-state tokamak reactor, in: Proceedings of the 18th Conf. on Fusion Energy, Sorrento, Italy October, 2000, (CDROM), pp. IAEA-CN-77/FTP2/14, IAEA, Vienna, 2001.

[4] K. Krieger et al., J. Nucl. Mat. 266 -269 (1999) 207

[5] W. Eckstein, R. Dohmen, A. Mutzke, R. Schneider. SDTrimSP: A Monte-Carlo Code for Calculating Collision Phenomena in Randomized Targets, IPP 12/3, 2007

[6] I. Bizyukov, A. Mutzke, R. Schneider, A. Gigler, K. Krieger. Nucl. Mater. Instr. B226 (2008) 1979-1986.

[7] I. Bizyukov, K. Krieger, N. Azarenkov, U. von Toussaint, J. Appl. Phys. 100 (2006) 113302.

[8] I. Bizyukov, K. Krieger, J. Appl. Phys. 101 (2007) 104906.

[9] I. Bizyukov, K. Krieger. J. Appl. Phys. 102 (2007) 074923.

[10] I. Bizyukov, J.W. Davis, A.A. Haasz. $\mathrm{CD}_{4}$ production from mixed W-C-D during simultaneous irradiation of $\mathrm{W}$ with $\mathrm{C}^{+}$and $\mathrm{D}^{+}$. This conference proceedings .

[11] K. Schmid, J.Roth, W.Eckstein, J. Nucl. Mater. 290-293 (2001) 148-152

[12] W.Eckstein, J.Roth, Nucl. Instrum. and Methods in Phys. Res., vol. B53 (1991) 279

[13] Sputtering by particle bombardment. Experiments and computer calculations from threshold to MeV Energies. Topics in Applied Physics. Vol. 110. Edited by R. Behrisch, W. Eckstein. ISBN 987-3-540-44500-5. Springer/Berlin/Heidelberg/New York: Springer-Verlag 2007. 


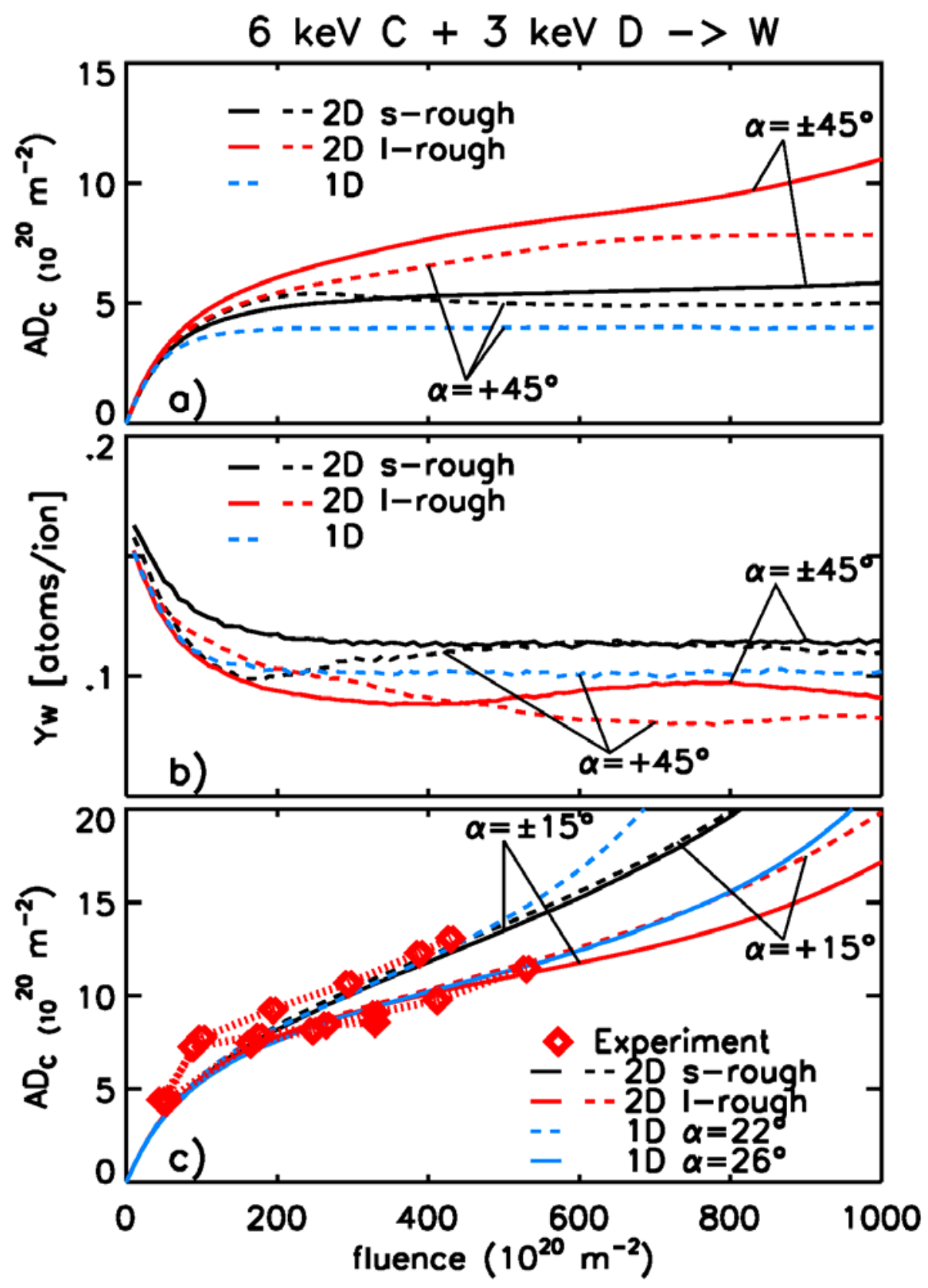

Figure 1. Evolutions of the areal density (AD) of C implanted (a,c) and W sputter yield (Y) (b) as a function of the fluence for impinging $3 \mathrm{keV} \mathrm{D}$ and $6 \mathrm{keV} \mathrm{C}$ ions on a W surface for different cases. Simulation results are shown as different lines. 1D SDTrimSP cases are marked as 1D, results obtained with SDTrimsSP-2D as 2D. The two different surface cuts from AFM (shown also in Figure 2) are labelled s-rough ('small roughness') and l-rough ('large roughness'). The geometry is indicated by a + for co-axial and \pm for symmetrical arrangements of the beams (the angle of incidence is also indicated). Experimental data are shown with open diamonds. 

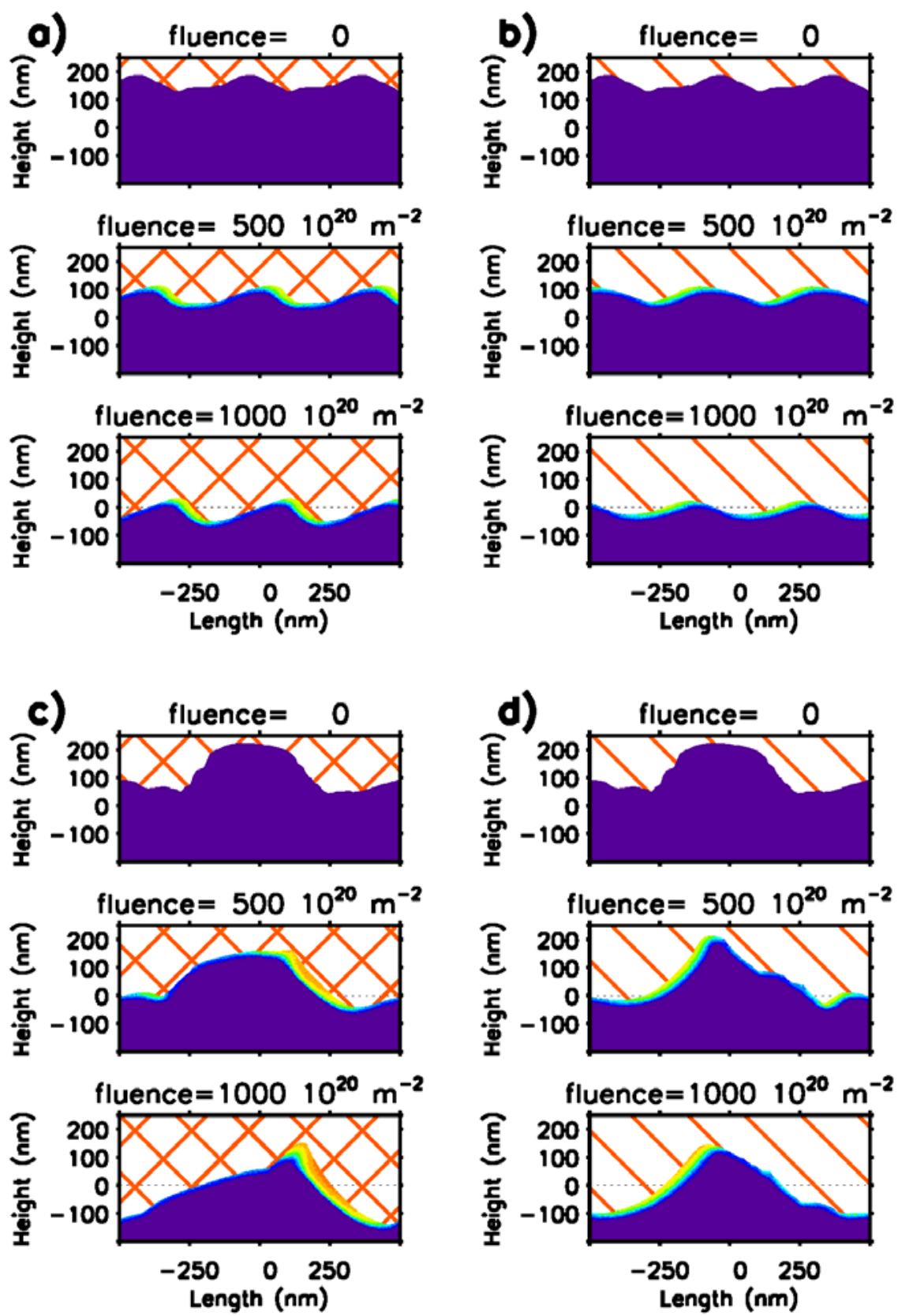

$\begin{array}{lllllllll}0.1 & 0.2 & 0.3 & 0.4 & 0.5 & 0.6 & 0.7 & 0.8 & 0.9\end{array}$

Rel. C (\%)

Figure 2. Evolution of the surface morphology under simultaneous bombardment with $6 \mathrm{keV} \mathrm{C}$ and $3 \mathrm{keV} \mathrm{D}$ ions for an angle of incidence of $45^{\circ}$. (a) and (c) - symmetrical bombardment of surface with low and high roughness correspondingly; (b) and (d) - coaxial bombardment of surface with small and large roughness, correspondingly. The lines show the trajectories of the projectiles before the collision with the surface. The colour corresponds to the relative $\mathrm{C}$ concentration. 